G. THE LUNAR INTERIOR 


\title{
LUNAR MAGNETIC FIELD MEASUREMENTS, ELECTRICAL CONDUCTIVITY CALCULATIONS AND THERMAL PROFILE INFERENCES
}

\author{
D. S. COLBUR N \\ Ames Research Center, NASA, Moffett Field, California, U.S.A.
}

\begin{abstract}
Steady magnetic field measurements of magnitude 30 to $100 \gamma$ on the lunar surface impose problems of interpretation when coupled with the non-detectability of a lunar field at 0.4 lunar radius altitude and the limb induced perturbations of the solar wind reported by Mihalov et al. at the Explorer orbit. The lunar time varying magnetic field clearly indicates the presence of eddy currents in the lunar interior and allows calculation of an electrical conductivity profile. The problem is complicated by the day-night asymmetry of the Moon's electromagnetic environment, the possible presence of the TM mode and the variable wave directions of the driving function. The electrical conductivity is calculated to be low near the surface, rising to a peak of $6 \times 10^{-3} \Omega^{-1} \mathrm{~m}^{-1}$ at $250 \mathrm{~km}$, dropping steeply inwards to a value of about $10^{-5} \Omega^{-1} \mathrm{~m}^{-1}$, and then rising toward the interior. A transition at $250 \mathrm{~km}$ depth from a high conductivity to a low conductivity material is inferred, suggesting an olivine-like core at approximately $800^{\circ} \mathrm{C}$, although other models are possible.
\end{abstract}

\section{Introduction}

The measurement of lunar magnetic fields has long been of interest because of the promise that these measurements would provide information about the lunar interior: a significant dipolar field would imply a dynamo action in the lunar interior similar to the Earth's dynamo, while the presence of induced fields would imply a value for the electrical conductivity in the lunar interior.

The first magnetic experiments were made by the U.S.S.R. on the Luna spacecraft. These set an upper limit on any lunar magnetic field and also indicated a magnetospheric region surrounding the Moon. More definitive measurements of the lunar environment came with the launching of the Explorer 35 lunar orbiting satellite in 1967. Magnetometers on this satellite detected no lunar bow shock and no evidence at periselene of a lunar field (Colburn et al., 1967; Ness et al., 1967). The upper limit for a lunar centered dipole moment was set at $10^{20} \mathrm{G} \mathrm{cm}^{3}$ corresponding to a maximum dipolar field component of $4 \gamma\left(1 \gamma=10^{-5} \mathrm{G}\right)$ on the lunar surface (Behannon, 1968). Larger surface fields were not ruled out if they were of quadrupole or higher order. Definitive measurements of lunar fields commenced with the deployment of the Lunar Surface Magnetometer on Apollo 12 and the Lunar Portable Magnetometer on Apollo 14.

In this paper we discuss first the measurement of a steady magnetic field at three points on the lunar surface. We consider next the predictions of other lunar magnetic regions inferred from satellite observations. Finally we consider the calculations of the electrical conductivity profile of the lunar interior determined by the Moon's electromagnetic response, and its implications for a thermal model. 


\section{The Instruments}

Explorer 35 was placed into lunar orbit on July 19, 1967 and is still in operation. The periselene is at an altitude of $0.4 R_{\mathrm{M}}\left(R_{\mathrm{M}}=1740 \mathrm{~km}\right)$ with the aposelene altitude $4.4 R_{\mathrm{M}}$ The orbital plane is tilted $11^{\circ}$ out of the ecliptic plane. Among the experiments are a plasma probe and two magnetometers. The Ames magnetometer that supplied the data reported here, is a three-axis vector fluxgate with $0.4 \gamma\left(1 \gamma=10^{-5} \mathrm{G}\right)$ resolution employing spin demodulation and filtering to avoid aliased data (Colburn et al., 1967; Mihalov et al., 1968). In this system the high bandwidth signals from the two sensors in the spin plane of the spinning spacecraft are multiplied by sines and cosines of the spin frequency and mixed appropriately to furnish a high bandwidth set of the three components of the vector field in an inertial (nonspinning) frame (Sonett, 1965). These are filtered to preserve the Nyquist criterion for alias free data before being sampled at the sampling rate (1 vector per $6.14 \mathrm{~s}$ ) and telemetered to Earth (Sonett, 1968). The maintenance of spectral purity is vital to the use of power spectral density estimates in determining an electrical conductivity profile.

The Apollo 12 Lunar Surface Magnetometer (LSM) is a triaxial fluxgate emplaced on the lunar surface at the Apollo 12 site at the eastern edge of Oceanus Procellarum, coordinates $3.0^{\circ} \mathrm{S}, 23.4^{\circ} \mathrm{W}$. The instrument telemeters to Earth a vector measurement of the field at a rate of 3.3 samples per s. The site survey mode that was commanded three days after emplacement rotated the sensors to each of the three coordinate directions in turn. The three sensors are mounted at the ends of three mutually orthogonal $100 \mathrm{~cm}$ booms, permitting a measurement of the local field gradient in the horizontal plane (Dyal et al., 1970b). Since no measurable gradient was found, a lower limit was placed on the distance to the field source, the distance also depending on the source configuration. A similar Lunar Surface Magnetometer was emplaced at the Apollo 15 site, with a third one scheduled for Apollo 16.

At the Apollo 14 site the field was measured by the Lunar Portable Magnetometer (LPM). This instrument was set up at two locations by the astronauts who then sent back the readings by voice channel. A $20 \mathrm{~s}$ time constant filter averaged out the high frequency fluctuations to measure the steady state field component at the site. A similar Lunar Portable Magnetometer is scheduled to be operated on Apollo 16.

Another magnetic measurement of the Moon has become possible by the magnetometer aboard the subsatellite launched into lunar orbit by the Apollo 15 command module (P. J. Coleman, Jr., principal investigator). It is expected this instrument and the similar one on Apollo 16 will be able to map locally magnetized areas on the lunar surface when on the lunar dark side or in the Earth's magnetic tail. The low orbital altitude, typically $100 \mathrm{~km}$ or less, will allow detection of surface feature at detail unobtainable at the orbit of Explorer 35. The Explorer measurement is very important in providing a measurement of the background field surrounding the Moon and is essentially unperturbed by the Moon's presence except when Explorer 35 is almost directly in the lunar shadow. 


\section{The Surface Field}

Data from the Apollo 12 LSM showed a steady field component at the site of $38 \gamma$ and it was known at the outset that this was not due to a dipole centered in the Moon, for a centered dipole of such strength would have been observed at the orbit of Explorer 35. It likewise could not have been due to a dipole source closer than $200 \mathrm{~m}$ to the instrument because of the null result of the gradient measurement. The possibility of a localized surface field highly variable with location suggested magnetization of the lunar crustal regions and prompted the design and approval of the LPM that made its measurements only slightly more than one year after inception.

Table I shows the vector field measurements reported for the two Apollo 14 sites

TABLE I

Magnetic field measurements at lunar sites (Dyal et al., 1971)

\begin{tabular}{|c|c|c|c|}
\hline Site & Apollo 12 & Apollo 14-A & $14-\mathrm{C}^{\prime}$ \\
\hline \multicolumn{4}{|l|}{ Coordinates } \\
\hline $\mathrm{S}$ & $3.0^{\circ}$ & $3.7^{\circ}$ & $3.7^{\circ}$ \\
\hline W & $23.4^{\circ}$ & $17.5^{\circ}$ & $17.5^{\circ}$ \\
\hline Location & LSM site & $170 \mathrm{~m}$ from $\mathrm{LM}$ & Cone crater rim \\
\hline $\begin{array}{l}\text { Field } \\
\text { Magnitude }\end{array}$ & $38 \pm 3 \gamma$ & $103 \pm 5 \gamma$ & $43 \pm 6 \gamma$ \\
\hline $\begin{aligned} \text { Components Up } \\
\text { East } \\
\text { North }\end{aligned}$ & $\begin{array}{l}-24.4 \pm 2.0 \gamma \\
+13.0 \pm 1.8 \gamma \\
-25.6 \pm 0.8 \gamma\end{array}$ & $\begin{array}{l}-93 \pm 4 \gamma \\
+38 \pm 5 \gamma \\
-24 \pm 5 \gamma\end{array}$ & $\begin{array}{l}-15 \pm 4 \gamma \\
-36 \pm 5 \gamma \\
-19 \pm 8 \gamma\end{array}$ \\
\hline Gradient & $<133 \gamma / \mathrm{km}$ & $>54 \doteq 7 \gamma / \mathrm{km}$ & \\
\hline
\end{tabular}

and the Apollo 12 site (Dyal et al., 1971; Dyal et al., 1970a). The vectors are also displayed in Figure 1. The Apollo 12 and 14-C' (Cone Crater) magnitudes are approximately $40 \gamma$ while the other Apollo 14 site has a magnitude factor of 2.5 greater. The vectors point down and southerly: the maximum angle between any two is $84^{\circ}$. The lower limit on the field gradient at Apollo 14 is defined as the average gradient between the two sites or $54 \pm 7 \gamma / \mathrm{km}$, while the upper limit for Apollo $12,133 \gamma / \mathrm{km}$ is determined by the site survey measurement, over a distance span on the order of $1 \mathrm{~m}$.

Models for the steady fields have been constructed but none have been completely satisfactory. Since it is known from Explorer 35 measurements that the surface field caused by any centered dipole must be less than $4 \gamma$, more localized field sources are hypothesized, which would presumably rule out the mechanism of a planetary dynamo. Alternatively, the measurements could represent the bulk magnetization of surface rocks. The remanent field in a rock slab which has been uniformly magnetized has a highly nonuniform field, the magnitude being greatest near the edges and direction altering radically with position near the edges. The magnitude becomes relatively 


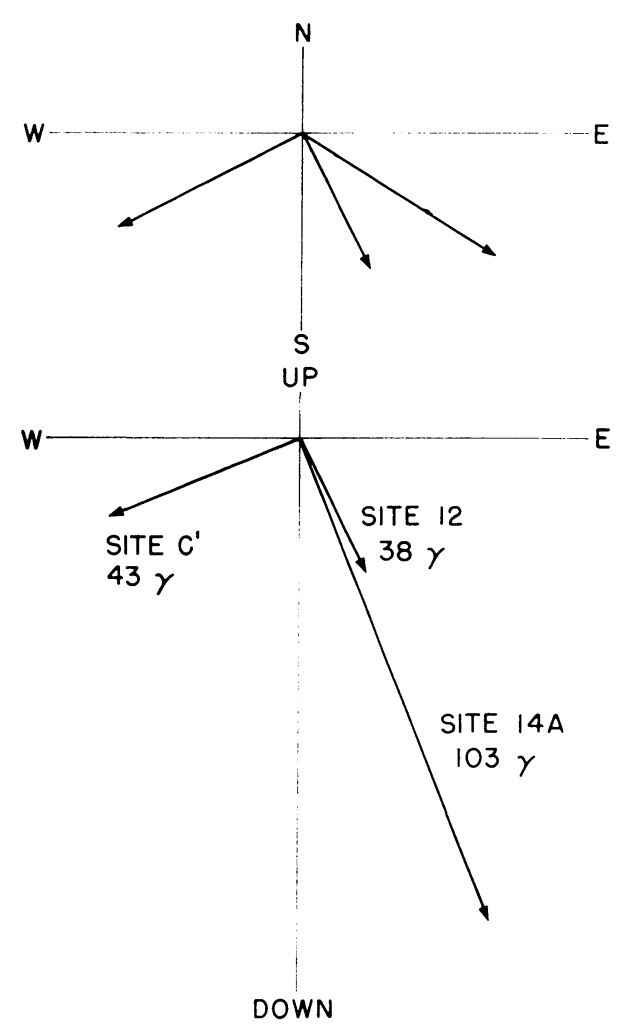

Fig. 1. Steady magnetic field vector at three locations on the Moon. Site 12 is the Apollo ALSEP location; sites $14 A$ and $C^{\prime}$ are the two measuring sites on the Apollo 14 moonwalk. The maximum angle between any two vectors is $84^{\circ}$. (From Dyal et al., 1971).

smaller as one approaches the broad flat region of the magnetized slab. The edge delineated by Cone Crater, as pointed out by Dyal, or perhaps of a larger Mare region hidden by surface features (Runcorn and Quaide, private communication) would explain the large difference in the two Apollo 14 measurements. In view of these differences and the proposed models, the significance of all measurements lying in a single quadrant is not clear. More data would be required to establish the direction of the original magnetizing field.

Remanent magnetism in rock areas consistent with magnetic measurements on Apollo rock can explain the steady field measurements (Runcorn et al., 1970). This implies, however, that large rock volumes cooled through the Curie point under the influence of a field of more than $1000 \gamma$. This creates difficulty in lunar thermal history models, as will be discussed later.

\section{Magnetic Map Inferred from Explorer 35}

Mihalov et al. (1971) have found perturbations in Explorer 35 magnetometer measure- 
ments that appear to map the lunar surface. To describe the method, it is necessary to review the magnetic environment at the Explorer 35 orbit.

The interplanetary magnetic field has a magnitude on the order of $5 \gamma$ but can become several times greater under disturbed conditions. The preferred direction is outward or inward along an Archimedes spiral determined by the solar wind and the Sun's rotation (Parker, 1963) although the field can assume any direction from time to time. The distinction between outward and inward can be made statistically, and for the period under question generally encompasses one outward and one inward sector (occasionally more) every solar rotation period of 27 days (Wilcox and Colburn, 1970). The solar wind is not deflected significantly by the Moon, as evidenced by the lack of a measurable bow wave, and hence the particles are believed to strike the lunar surface and be neutralized there, while directly behind the Moon is a cavity in which solar wind is essentially absent and the magnetic field is slightly enhanced because of the diamagnetism of the solar wind (Colburn et al., 1971). At the border of the cavity a characteristic dip in magnetic field magnitude is observed. Beyond the Mach angle defining the rarefaction wave, however, a peak in the magnetic field is occasionally observed. These are believed to be due to a lunar solar wind interaction occurring at the limb and propagating outward along the Mach angle. The mechanism is postulated to be the deflection of solar wind by local magnetic field. No magnetic effect of the Moon is seen over the large majority of the Explorer 35 orbit lying forward of this Mach angle; the magnetic field is as if measured from a spacecraft very far from the Moon. Because the solar wind travels at a speed faster than known magnetosonic and Alfvenic propagation speeds, the information signaling the presence of the Moon cannot travel upstream to these regions. From time to time the magnetic field peak exterior to the rarefaction wave becomes unusually large. There were 100 large peaks representing $7.4 \%$ of the possible times of occurrence. Mihalov has catalogued these occurrences and related them to regions on the Moon at the limb by associating each occurrence with the point on the lunar surface closest to the solar wind velocity vector

\section{TABLE II}

Regions on the lunar surface where the concentration of source locations was tested (Mihalov et al., 1971). $F$ is the fraction of observations showing the anomaly and $P$ is calculated probability that there is no concentration at the location. The observations are from the orbiting Explorer 35 satellite. Mihalov et al. conclude that local magnetization is the favored explanation for the phenomena.

\begin{tabular}{|c|c|c|c|c|c|}
\hline $\begin{array}{l}\text { Seleno- } \\
\text { centric } \\
\text { latitude }\end{array}$ & $\begin{array}{l}\text { Seleno- } \\
\text { centric } \\
\text { longitude }\end{array}$ & $\begin{array}{l}\text { Area } \\
\left(\mathrm{X} \mathrm{10} 0^{5} \mathrm{~km}^{2}\right)\end{array}$ & $F$ & $P$ & Nearby future \\
\hline $5^{\circ}-20^{\circ} \mathrm{S}$ & $135^{\circ} \mathrm{E}-165^{\circ} \mathrm{W}$ & 10 & 0.5 & $\ll 10^{-5}$ & Mare X (Gagarin) \\
\hline $6^{\circ} \mathrm{N}$ & $88^{\circ} \mathrm{W}$ & 2 & 0.4 & $<10^{-5}$ & 'Montes d'Alembert' \\
\hline $0^{\circ}-20^{\circ} \mathrm{N}$ & $60^{\circ}-115^{\circ} \mathrm{E}$ & 10 & 0.3 & $3 \times 10^{-3}$ & Mare Marginis \\
\hline $5^{\circ} \mathrm{S}$ & $138^{\circ} \mathrm{W}$ & 0.7 & 0.3 & $6 \times 10^{-3}$ & Crater 244 (Vavilov) \\
\hline $2^{\circ} \mathrm{N}$ & $35^{\circ} \mathrm{W}$ & 1 & 0.2 & $<10^{-5}$ & Eneke-Kepler \\
\hline $5^{\circ} \mathrm{N}$ & $0^{\circ}$ & 0.9 & 0.1 & $5 \times 10^{-4}$ & Pallas \\
\hline $0^{\circ}$ & $25^{\circ} \mathrm{E}$ & 0.8 & 0.1 & $4 \times 10^{-5}$ & Delambre \\
\hline
\end{tabular}


drawn through the spacecraft location at the time of the observation (Mihalov et al., 1971; Sonett and Mihalov, 1972). The mapping has been shown to be statistically significant. In Table II are shown the areas outlined by Mihalov as being connected with the exterior peaks observed by Explorer 35 .

The table shows that the highlands are favored, and also that the far side is favored. While mechanisms other than local magnetic fields could be postulated as causes of the field increases seen by Explorer 35, Mihalov et al. find cogent reasons for believing that the local magnetic field is the most likely cause. On the theoretical side, Barnes et al. (1971) have investigated the possible frequency of magnetic structure on the surface. They find that regions of field such as indicated by the Explorer 35 perturbations must have a length scale of at least $10 \mathrm{~km}$ and a compressed field strength of more than $10 \gamma$, and that thousands of these could be present on the lunar surface.

\section{Electrical Conductivity Profile Calculations}

The lunar surface magnetic field measurement was early thought of as a means of probing the interior of the Moon (Sonett, 1966). If we consider the Moon as a sphere whose electrical conductivity is a function of radius only, we find that there are two modes in which it can respond to the time varying electrical and magnetic field associated with the solar wind: the transverse magnetic (TM) and the transverse electric (TE) (Schubert and Schwartz, 1969). For the TM response the driving function is an electrical field due to the solar wind. Because of the high electrical conductivity of the plasma constituting the solar wind, the electric field must be considered to be zero in a frame at rest in the solar wind plasma. A simple transformation to a frame at rest with respect to the moon shows an electric field of $E=$ $v \times B$ where $B$ is the interplanetary magnetic field and $v$ is the velocity of the Moon with respect to the solar wind. This response should extend all the way down to zero frequency. The current system of the response must be such that the current flows through the Moon and its crust and closes through the highly conducting solar wind. If this response were to be significant, it should cause a bow shock; a phenomenon not observed by Explorer 35. It is generally concluded that the relatively high resistance of the Moon's crustal layers effectively cuts off the TM mode (Sonett and Colburn, 1967). The high resistance is deduced from the probable electrical conductivity of lunar material at the mean surface temperature, which must be assumed by material more than a few meters deep considering the low thermal conductivity of rock materials. An exhaustive statistical analysis of magnetometer measurements may yet show a modicum of TM response, but it must remain a secondary effect.

We turn now to the TE mode, which is excited by $\dot{B}$, the rate of change of the interplanetary magnetic field. The response of a planetary body to this mode is the establishment of eddy currents within the body that do not need to flow through the relatively nonconducting crust. This mode does not occur in the steady state but is observable at frequencies commensurate with lunar magnetic field measurements. Preliminary findings on the TE response of the Moon were reported by Sonett et al. (1971b, c). 


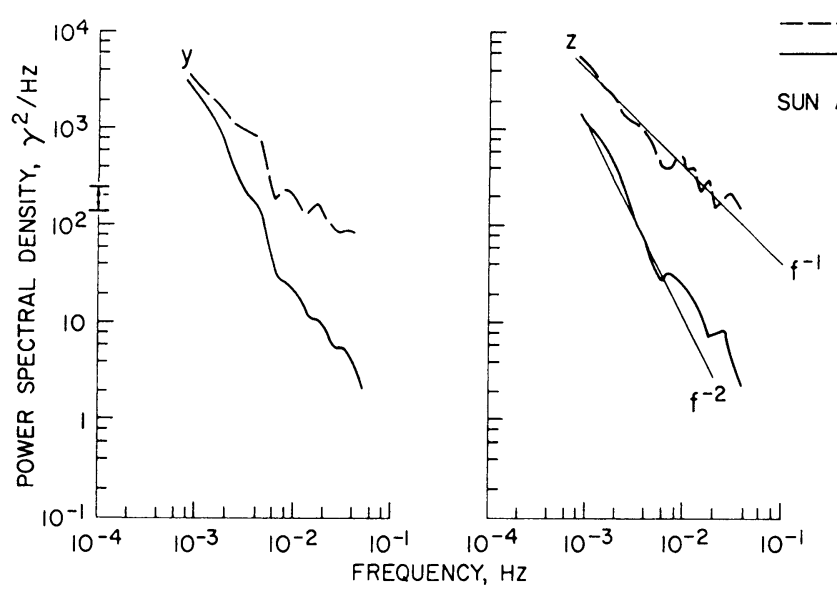

Fig. 2. Power spectral density of the interplanetary field near the Moon (Explorer 35) and the lunar surface field (Apollo 12) for the same 2-h time period. The two horizontal components are shown: $Z$ is north and $Y$ is east at the Apollo 12 site. Other 2-h segments show variations from this example but all show an amplification in the horizontal components increasing with frequency.

Figure 2 shows a typical plot of power spectral density versus frequency for the horizontal components of the lunar surface field (Apollo 12) and for the same time period the corresponding components of the free stream solar wind field near the Moon. The time period was $2 \mathrm{hr}$ and the Apollo 12 magnetometer location $33^{\circ}$ from the Moon-Sun line. The Moon was outside the Earth's bow shock in the solar wind. The power in the interplanetary field drops off with frequency approximately as $f^{-2}$, a typical frequency dependence for interplanetary spectra. The power in the surface components is larger, particularly at the higher frequencies.

Autocorrelation techniques instead of cross correlation techniques are used because of the complication of the variable Doppler shift between the locations of the two measurements. The analysis is based on the assumption that the free stream field is the sole driving function for the lunar response. This is made credible by the examination of the amplification of particular sine wave cycles or transients such as were published in Sonett et al. (1971c).

Data from the first lunation were examined to find periods in which both magnetometers were reading simultaneously with no data gaps. Seven 2-h and seven 1-hr segments were used, with the Explorer 35 data transformed into LSM coordinates. The Apollo 12 data were filtered and decimated, to approximate the same filter and bit rate as the Explorer 35 data. Autocorrelation and power spectral density estimates were made for several frequencies. For the 2-hr swaths 50 frequencies were used, linearly spaced from $0.83 \mathrm{mHz}$ to $41.5 \mathrm{mHz}$. For the 1 -h swaths the number of frequencies was 25 (1.66 to $41.5 \mathrm{mHz}$ ) - The amplification $A$ was defined as the square root of the ratio of power density at the lunar surface to power density in the free stream. The subscript $x, y$, or $z$ is added to signify respectively the vertical, east, and north components of the vectors as viewed from Apollo 12. 


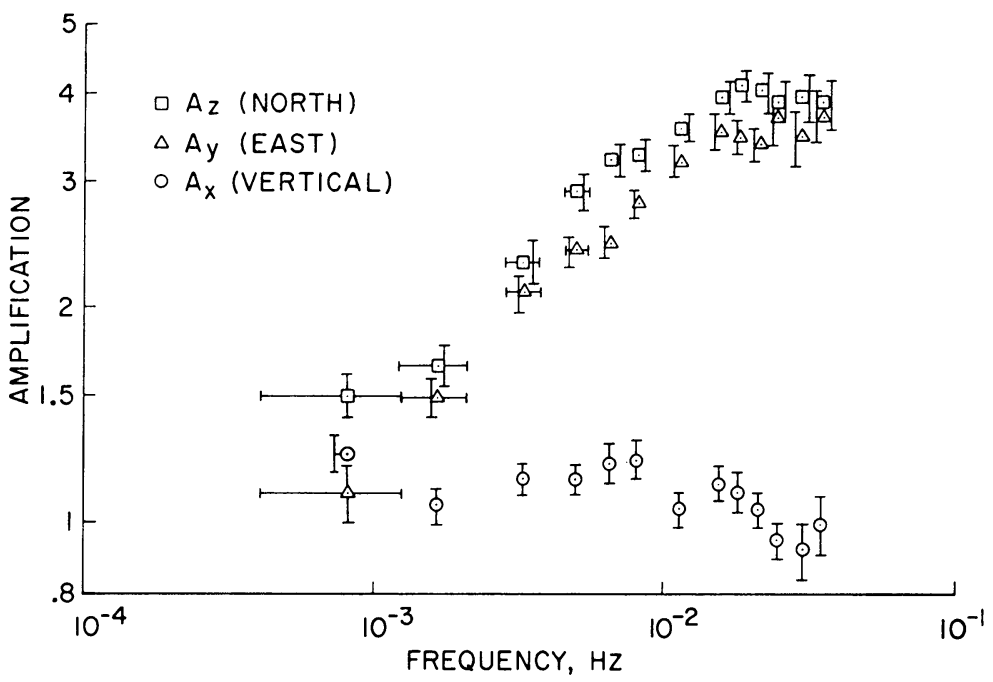

Fig. 3. Amplification, the ratio of amplitudes of lunar surface and free stream magnetic fields, shown as a function of frequency. The amplification is defined as the square root of the ratio of power spectral densities. The error bars for frequency are the windows defined by the lags in the autocorrelation calculation. The error bars in amplification are the one standard deviation limits determined from the means of 14 data spectra. Amplification occurs for the north and east components but remains near unity for the vertical component.

Values of $A_{x}, A_{y}$, and $A_{z}$ are shown in Figure 3 at representative frequencies common to the 14 cases. The averages of 14 values are plotted with error bars designating the one standard deviation error in the mean. (The point at $8.3 \mathrm{mHz}$ is an average of only seven cases.) The amplification for the horizontal components starts near unity at the lowest frequency and rises significantly with frequency to values approaching 4 at the highest frequencies. For the vertical component the amplification remains near unity.

The results in Figure 3 were anticipated by theoretical analyses. Blank and Sill (1969) derived a model for the TE mode and Schubert and Schwartz (1969) derived a model dealing consistently with the combined TE and TM modes in a wave field. In both treatments a thin current layer was assumed to surround the Moon such that outside of the current layer the field had the free stream value, the normal component of which (since $\nabla \cdot B=0$ ) was conserved across the layer. The approximation is appropriate on the sunlit side where the solar wind plasma is moving into the Moon at a speed both supersonic and super Alfvenic, so that in the magnetohydrodynamic approximation no information regarding the Moon's presence may be conveyed upstream. The confinement permits very large amplifications of horizontal components. The current layer is not appropriate on the dark side; however, model calculations show that measurements on the sunlit side are not altered more than a few percent by the dark side portion of the assumed current system, and the symmetry is necessary for tractibility.

Attenuation of the vertical component at a given frequency would be substantially 
complete at a depth where the electrical skin depth for that frequency is small. If the Moon were immersed in a vacuum, the attenuation would drop off as $r^{3}$ and be observed at the surface. The near unity values for the vertical amplification imply that a current layer is indeed present above the lunar surface and much closer than the underlying volume of high electrical conductivity.

The scatter in the $A_{x}$ data has not yet been satisfactorily explained but may be due to plasma noise effects and departure from spherical symmetry.

The magnetic field amplification at the lunar surface, observed experimentally, is believed to give information about the electrical conductivity in the lunar interior. The Moon is considered here to have an electrical conductivity $\sigma(r)$ varying only with radial distance. The magnetic field oscillation in the solar wind is defined as

$$
H=\hat{\eta} H_{0} \exp \left\{2 \pi i\left(\frac{\zeta}{\lambda}-f t\right)\right\}
$$

The cartesian coordinate system $(\xi, \eta, \zeta)$ with unit vectors $\hat{\xi}, \hat{\eta}, \hat{\zeta}$ is fixed relative to the Moon, which moves through the solar wind in the negative $\zeta$ direction with speed $v$ relative to the wave front. (For transverse waves, neglecting propagation speeds, $v$ is the aberrated solar wind speed.) The amplitude is $H_{0}$, the wavelength $\lambda$, and the frequency of the oscillation is $f$. In the lunar interior the solution of Maxwell's equations for the TE mode is represented by a potential $\Omega$ satisfying

$$
\nabla^{2} \Omega+k^{2} \Omega=0
$$

where

$$
k^{2}=4 \pi^{2} f^{2} \mu \varepsilon+i 2 \pi f \mu \sigma
$$

and $\mu$ and $\varepsilon$ are taken as free space values. The potential is found as a sum of product solutions of the form

$$
\Omega=\sin \phi \sum_{l=1}^{\infty} F_{l}(r) \varrho_{l}^{1}(\cos \Theta)
$$

where $r, \Theta$, and $\phi$ are spherical polar coordinates with $\zeta$ the polar axis and $\varrho_{l}^{1}$ are associated Legendre polynomials. The boundary condition for the TE mode is the matching of the lunar surface normal component of the magnetic field to the free stream value, as discussed earlier, confining the lunar disturbance to the immediate proximity of the Moon.

Equations for the calculation of the fields are given elsewhere (Sonett et al., 1971d); alternatively they may be written for the TE case by taking the solution to the Earth induction problem (Lahiri and Price, 1939, or see Chapman and Bartels, 1962, Section 22.13, Equations 23-31) and making proper modifications for the changed boundary conditions. For the work reported here, the transfer function is defined as the ratio of the magnitudes of transverse components for the first mode of the modal expansion.

Using the values of $A_{y}$ and $A_{z}$ averaged from 14 swaths (of 1- and 2-h duration) Sonett et al. have calculated a curve of amplification versus frequency. Because of the 
significant differences in $A_{y}$ and $A_{z}$, not yet explained, fits were made separately for $A_{y}^{2}, A_{z}^{2}$, and $\bar{A}=\left[0.5\left(A_{y}^{2}+A_{z}^{2}\right)\right]^{1 / 2}$.

The inversion method consists of starting with a conductivity profile, calculating an $A$ versus $f$ curve and minimizing by the least squares method the deviation of the calculated $A$ with $\bar{A}, A_{y}$, or $A_{z}$. In order to do this the conductivity profile was characterized by eight parameters, namely the conductivity at $r=800,1200,1400,1450,1490$, 1510,1550 , and $1740 \mathrm{~km}$. The conductivity for $0 \leqslant r \leqslant 800 \mathrm{~km}$ was considered constant, the method not being very sensitive to conductivities at these depths. At all other values of $r, \log r$ is defined as a linear interpolation from neighboring $\log r$ values. In each iteration the amplification for the model is calculated at eight frequencies, namely $0.83,1.7,5,12,17,22,25$, and $35 \mathrm{mHz}$, and the least squares fit at those frequencies between the model and the data is minimized by successive computer iterations using the Newton Raphson method.

The method showed significant convergence in five iterations. Figure 4 shows the resulting fit of a model amplification curve in which the rms value $\bar{A}$ was fitted. Figure 5 shows the conductivity profile found by the routine that produces this result and profiles found by matching $A_{y}$ or $A_{z}$ independently. In each case the profile is found to have a maximum near $r=1500 \mathrm{~km}$. The conductivity starts at a low value at the surface as is expected because of the mean temperature of $-30^{\circ} \mathrm{C}$ and the strong temperature dependence of candidate lunar materials. Because the current system is effectively cut off, the method is imprecise at low conductivities. The conductivity then rises with depth to a maximum of about $6 \times 10^{-3} \Omega^{-1} \mathrm{~m}^{-1}$ at $r=1500 \mathrm{~km}$ and then decreases by some 2 orders of magnitude in $100 \mathrm{~km}$ before resuming a rise with depth.

The temperature profile can be inferred from the conductivity profile only indirectly,

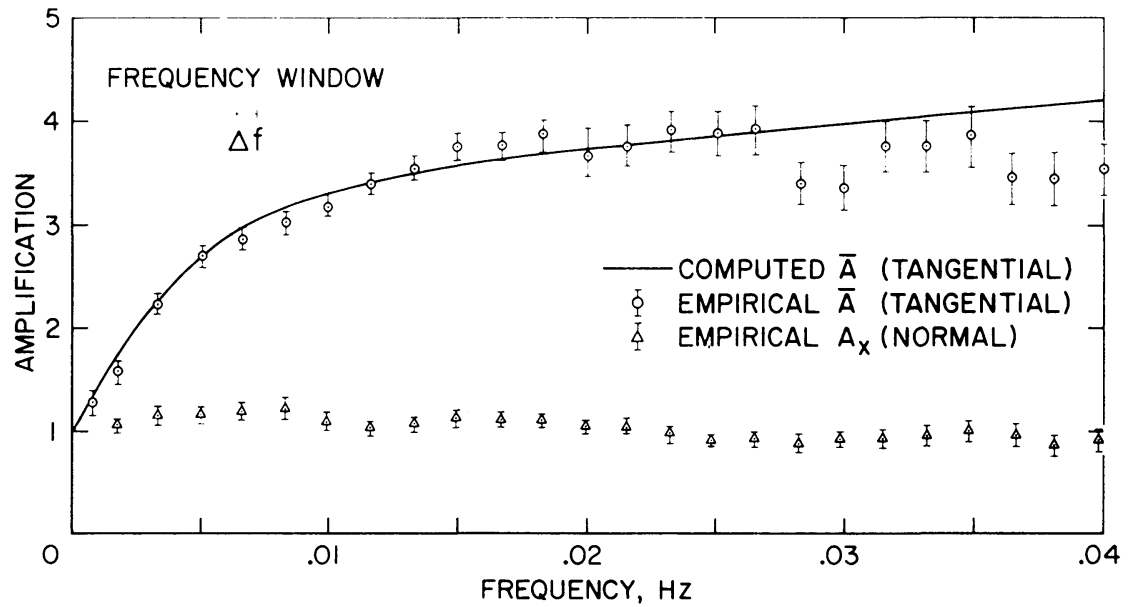

Fig. 4. Amplification as a function of frequency. The data are as in Figure 3, with the tangential amplifications combined by the relation $\bar{A}=\left[0.5\left(A_{y}^{2}+A_{z}^{2}\right)\right]^{1 / 2}$. The solid line is the amplification calculated from a model described in the text. The amplification of the model is fitted to $\bar{A}$ values at frequencies of $0.83,1.7,5,12,17,22,25$, and $35 \mathrm{mHz}$. 


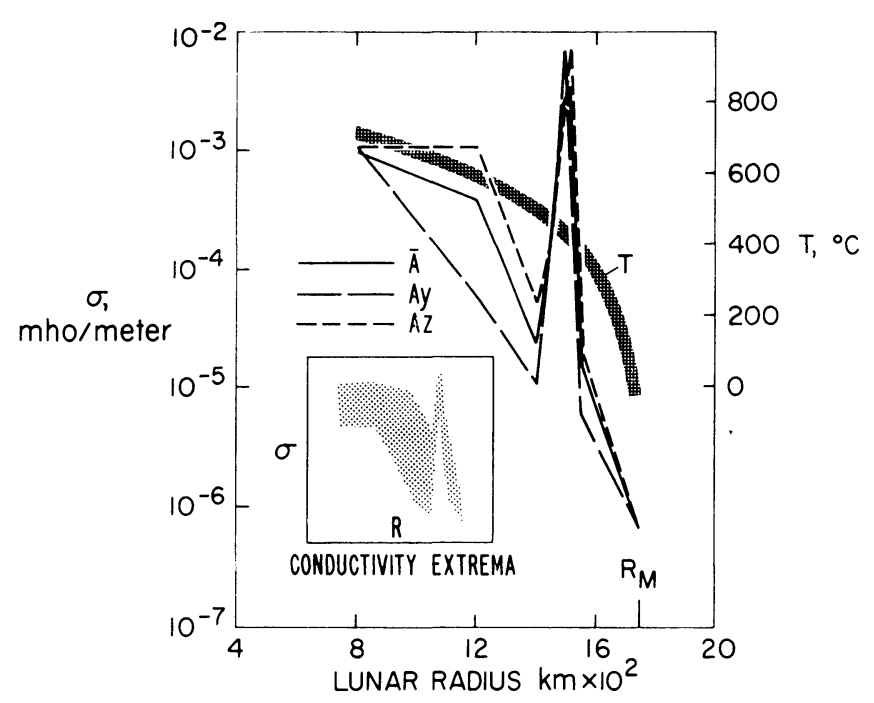

Fig. 5. Lunar electrical conductivity as a function of radial distance, according to calculations based on the amplifications $A_{y}, A_{z}$, and $\bar{A}$. As depth increases the conductivity rises to a peak at $r=1500 \mathrm{~km}$, decreases to a minimum at $r=1400 \mathrm{~km}$ and then continues to rise. Six other cases were run, using the one standard deviation limits of the As for calculating the conductivities; these all contain the peak and lie in the shaded area shown. Plotted also is a tentative version of a lunar thermal profile. The assumption of a relatively smooth thermal profile and known conductivity functions requires a core conductivity similar to that of olivine with a transition to a more highly conducting material at $r=1500 \mathrm{~km}$.

since it also depends on composition and conductivity-temperature functions. If candidate materials are ordered according to conductivity, one of the more conducting is a basalt such as the Lunar Sample 10024.22 analyzed by Nagata et al. (1970; see also Schwerer et al., 1971). One of the less conducting is an olivine (see England et al., 1968). The conductivity gradient from the surface to $r=1500 \mathrm{~km}$ is approximated by a $2{ }^{\circ} \mathrm{C} / \mathrm{km}$ temperature gradient for the basalt function and a $4^{\circ} \mathrm{C} / \mathrm{km}$ gradient for the olivine, with most other candidate materials lying somewhere in between. It is not likely that the temperature can decrease with depth between $r=1500 \mathrm{~km}$ and $r=1400 \mathrm{~km}$ because any such gradient occurring during the Moon's formation would have smoothed out during the lunar history, and localized present-day heat sources at that depth are unlikely. If this reasoning is correct, the transition from 1500 to $1400 \mathrm{~km}$ must be accompanied by a modest increase in temperature as well as a large decrease in conductivity. The transition is then plausible if the conductivity function at $1500 \mathrm{~km}$ is relatively high, like that of a Nagata basalt, and the function at $r=1400 \mathrm{~km}$ is low, like that of an olivine. This reasoning leads to a moderate temperature profile such as sketched in Figure 5, with a temperature on the order of $700^{\circ} \mathrm{C}$ at $r=800 \mathrm{~km}$ and approximately $800^{\circ} \mathrm{C}$ at the center. Since the sensitivity of the model is low near the center, the central temperature is inferred from the fit of lunar thermal history models to the profile outside of the boundary $r=800 \mathrm{~km}$ 
(Fricker et al., 1967). The thermal profile curve is consistent with a lunar thermal history model with $25 \%$ chondritic radioactive concentration; i.e., one must assume that the Moon had been relatively deficient in radioactive concentration compared to the chondritic meteorites during its formation to avoid higher temperatures in the present epoch, assuming traditional values for thermal conductivity. Acceptance of a solid state thermal conduction mechanism such as proposed by Runcorn (1962; see also Turcotte and Oxburgh, 1969) would not alter the present-day thermal profile estimate but would allow the Moon to have been hotter at an earlier time.

In a parallel investigation using a different set of magnetic data Dyal and Parkin (1971) derived a monotonic lunar profile. The data were restricted to periods when the LSM was in the lunar night and consequently separated from the confining pressure of the incoming solar wind. Any lunar induced magnetic perturbation on the dark side is free to propagate into the essentially plasma-free cavity in the solar wind shadow. The geometry is complicated by the fact that the field direction is generally oblique to the axis of the shadow region. No unified model of the Moon's reaction that includes both the sunward confinement and the cavity region has been developed. For tractibility, just as Sonett et al. (1971a, d) assumed a spherical current layer for the dayside analysis, Dyal and Parkin assumed the Moon to be immersed in a vacuum, since the distance from the lunar measurement site to the confining currents is large.

Dyal and Parkin analyzed the vertical component of many step function transients observed in the dark side. They fitted the data to a two-layer model moon of radius $R_{\mathrm{M}}$, with $r=R_{1}$ denoting the boundary between the inner layer of conductivity $\sigma_{1}$ and the outer nonconducting layer. Theory predicts that if the vacuum field surrounding the model moon is homogeneous and undergoes a unit step increase, the vertical component of the surface field rises as (Dyal and Parkin, 1971)

$$
B=1-\frac{6}{\pi^{2}}\left(\frac{R_{1}}{R_{m}}\right)^{3} \sum_{s=1}^{\infty} \frac{1}{S^{2}} \exp \left(\frac{-S^{2} \pi^{2} t}{\mu_{0} \sigma_{1} R_{1}^{2}}\right)
$$

The initial value at $t<0$ is $B=0$, and for $t \gtrsim 0, B=1-\left(R_{1} / R_{\mathrm{M}}\right)^{3}$. The final value is unity. The dominant time constant is $\tau=\mu_{0} \sigma_{1} R_{1}^{2} / \pi^{2}$, controlling $\sim 60 \%$ of the series at the onset and dominating more and more as the higher order terms die out.

In Figure 6 is shown the data for one of the many steps analyzed by Dyal and Parkin; in this case the step was negative-going. The dashed line shows the fit of their model, with $0.95 R_{\mathrm{M}}<R_{1}, \sigma=1.5 \times 10^{-4} \Omega^{-1} \mathrm{~m}^{-1}$ and the time constant $\tau \sim 55 \mathrm{~s}$. The curve marked 'step response' is the response under the same conditions of a model similar to that of Figure 5, a single current layer profile in which the conductivity is considered negligible except at $r=1505 \mathrm{~km}$ where a layer of thickness $\Delta r$ has a conductivity $\sigma$ characterized by $\sigma \Delta r=70 \Omega^{-1}$. The initial fractional drop is $1-\left(R_{1} /\right.$ $\left.R_{\mathrm{M}}\right)^{3}$, for this model as well as for the two-layer model, and for $R_{1}=1505 \mathrm{~km}$ the initial drop does not conform to the data, as is shown in the figure. Schubert and Colburn (1971) suggest, however, that the input function is imperfectly known but 


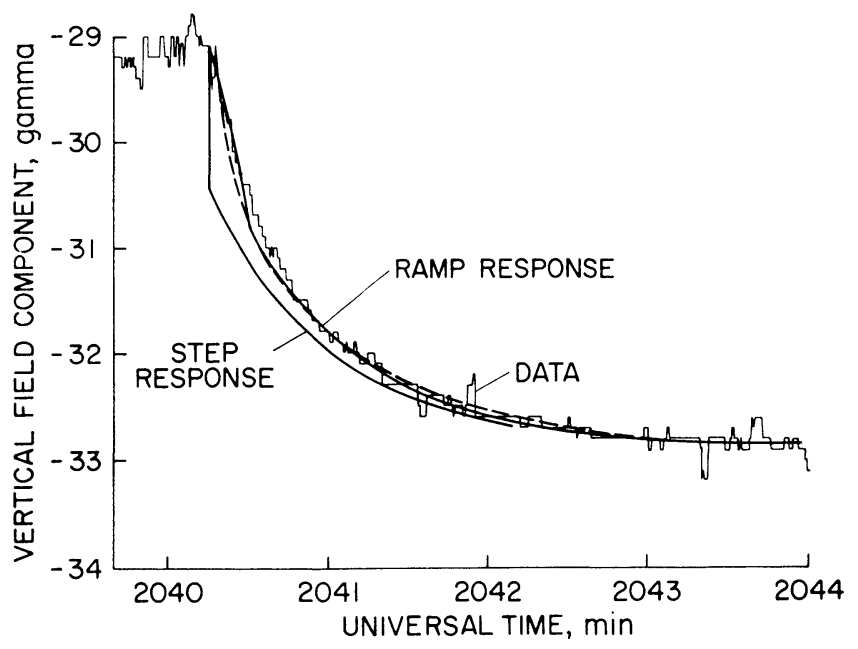

Fig. 6. Transient observed in the vertical component of lunar surface magnetometer data while the magnetometer site was in lunar night. Eddy currents in the interior inhibit rapid changes in this component. The dashed line is the response of the Dyal and Parkin model obtained from many of these transients (Dyal and Parkin, 1971). Another model, described in the text, has the responses shown depending on whether the input function is best described as a step or a ramp (Schubert and Colburn, 1971).

that since most transients in the solar wind are frozen into the plasma, the entire Moon does not see the field change at the same time. A more appropriate concept is that of a wave front sweeping over the Moon in a finite length of time. Schubert and Colburn approximate the effect of the moving wave front by a ramp function by which the surrounding field, assumed homogeneous, rises to its final value in $15 \mathrm{~s}$. For this driving function the current layer model responds according to the curve labeled 'ramp response', which is satisfactorily close to the data.

The data of Figure 6 represent but one case of the 10 used by Dyal and Parkin to obtain a size and conductivity for the core: $\sigma=1.7 \pm 0.4 \times 10^{-4} \Omega^{-1} \mathrm{~m}^{-1}, R_{1}>$ $0.95 R_{\mathrm{M}}$. Consequently, while a fit to the data of Figure 6 is not definitive Schubert and Colburn make two points: (1) it is important to consider the correct driving function for the Moon, and (2) if the ramp approximation is valid, the profile of Figure 5 and also two-layer profiles with $R_{1}<0.95 R_{\mathrm{M}}$ are candidates for fitting the transient data.

Dyal and Parkin (1971) report a long tail on their transient response curves representing time decays longer than $4 \mathrm{~min}$. The fit to these data implies a region of still higher conductivity deeper in the Moon, represented in the model by a third layer of conductivity $\sigma_{2}>10^{-2} \Omega^{-1} \mathrm{~m}^{-1}$ and $R_{2}>0.6 R_{\mathrm{M}}=1040 \mathrm{~km}$. The implications for the thermal profile are a higher inner temperature, as high as $1240 \mathrm{~K}=967^{\circ} \mathrm{C}$.

It has been questioned whether the conductivity maximum in the conductivity profile is necessary to fit the data because a monotonic profile is simpler and would appear to follow the experimental data fairly closely. A comparison of the fits of 


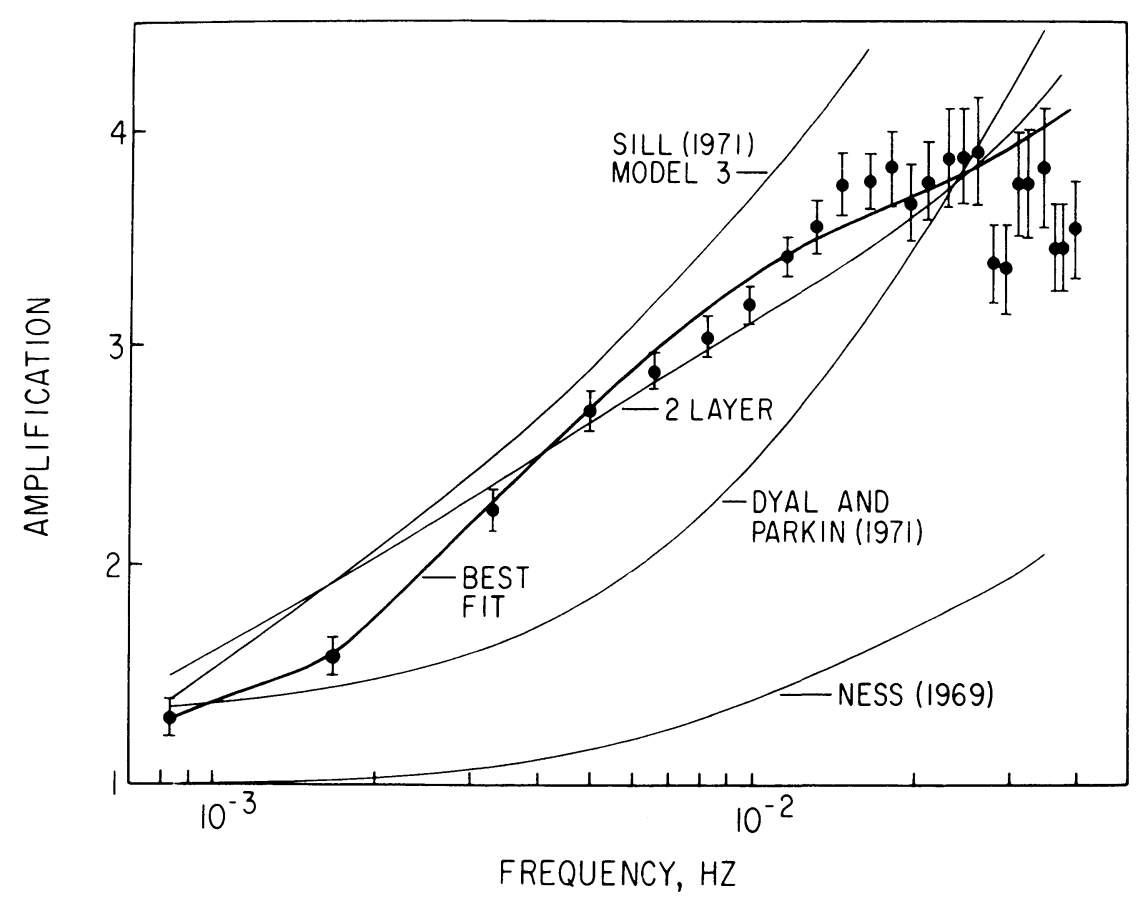

Fig. 7. Amplification as a function of frequency for several lunar electrical conductivity models. The data and the curve labeled 'best fit' are as shown in Figure 4. The curve labeled 'two-layer' is a best fit under the constraints of a two-layer model when $R_{1}=1560 \mathrm{~km}$. The conductivity peak in the model is necessary in order to come close enough to the three lowest frequency data points.

various models is shown in Figure 7. A two-layer model is shown, with a core of radius $R_{1}=1560 \mathrm{~km}$ and a conductivity $\sigma=7.6 \times 10^{-4} \Omega^{-1} \mathrm{~m}^{-1}$. This model was attained as a best fit to the $A$ data at the given radius and is close to the model of Kuckes (1971) for which $R_{1}=1580 \mathrm{~km}$ and $\sigma=6 \times 10^{-4} \Omega^{-1} \mathrm{~m}^{-1}$. The difference between the fits of the two-layer and the conductivity peak model is principally at the three lowest frequencies: a two-layer model fitting the middle range cannot dip sharply enough to approach the data at the lower frequencies. Other curves shown for comparison are those of model 3 of Sill (1971) and Ness (1969). Other models investigated by Sill do not provide a better fit than his model 3. The model of Ness had $R_{1}=1426 \mathrm{~km}$ and $\sigma=8 \times 10^{-5} \Omega^{-1} \mathrm{~m}^{-1}$. This model was based on an interpretation of a transient event observed by Explorer 35. Sonett et al. (1971e) have since proposed an alternate explanation for the data that would remove the basis for that model. It was concluded that Explorer 35 was too far from the Moon to measure a lunar response.

A difficulty with model fits for frequencies greater than $10^{-2} \mathrm{~Hz}$ lies in the wavelength of the driving function (Schubert and Schwartz, 1972). Convection in the solar wind dominates; wave propagation velocities in the frame of reference of the solar wind plasma are generally much less than the bulk velocity of the solar wind so 
that the wavelength can be approximated by

$$
\lambda=(n \cdot v) / f
$$

where $n$ is the unit normal to the wave front and $v$ the solar wind bulk velocity. For frequencies below $10^{-2} \mathrm{~Hz}, \lambda / R_{\mathrm{M}} \gg 1$ for nearly all wave directions. Model fits by Sill and Kuckes are based on infinite wavelength. For the work reported here, $n \cdot v$ is taken to be $400 \mathrm{~km} \mathrm{~s}^{-1}$, introducing differences in the high frequency response and also in the definition of the driving function using a modal expansion.

It is possible to extend the model to include the higher order spatial harmonics. The extended model, more accurate at high frequencies, has been presented by Schubert and Schwartz (1971). The use of the model at frequencies above $10^{-2} \mathrm{~Hz}$ will depend on the feasibility of sorting out correct wave vector directions in the experimental data.

\section{Conclusion}

Measurement of a steady field at three places on the lunar surface implies that the surface layer was magnetized during cooling by a field estimated to have been at least of $1000 \gamma$ amplitude (Helsley, 1970). The source of this magnetizing field is not clear (cf. Sonett and Runcorn, 1971). Had a dynamo once been active in the lunar interior, using normal values of thermal conductivity, the Moon would now possess a molten core. This appears unlikely from the electrical conductivity calculations, and also from rigidity arguments. If the field were an extension of the photospheric field, its value at the photosphere would be very large; under conditions where the field pressure dominated the solar wind, the field would decrease as $r^{-3}$, requiring $10^{5} \mathrm{G}$ at the photosphere. If the solar wind had sufficient momentum density to shape the magnetic field, the radial field would follow an $r^{-2}$ law (Parker, 1963). The field requirement would then be more modest, $600 \mathrm{G}$, but the solar wind density would be orders of magnitude greater than the present day value, a situation not expected to have occurred after a possible T-Tauri stage of solar evolution (Sonett et al., 1968). Polarity reversals due to the Sun's rotation would also make it unlikely that the Sun's field could magnetize a rock layer slowly cooling through the Curie point.

Immersion of the Moon in the Earth's field to attain a $1000 \gamma$ magnetizing field implies a synchronously rotating Moon at an orbital distance dangerously close to the Roche limit, unless the earth's field had been far greater than present day values (Dyal et al., 1970a). A satisfactory explanation for the required magnetizing field has not yet been found.

The conductivity profiles obtained by the dayside data imply a discontinuity at $r=1500 \mathrm{~km}$. The conductivity maximum implies a change at that depth from a more to a less electrically conducting material. Alternatively the presence at that depth of a relatively thin highly conducting layer as suggested by Urey (see Urey et al., 1971) would also fit the electrical conductivity data. In this case the $\sigma \Delta r$ product would be $\sim 10^{2} \Omega^{-1}$ and the lunar interior temperature might be lower than suggested if a rock other than olivine is postulated for the deep interior. 
The barrier at a depth of some $250 \mathrm{~km}$ appears even for models with monotonic conductivity profiles, although there is some question from the darkside transient data as to whether a shallower depth is more appropriate. A barrier implies a change of composition or state at that depth, possibly the lower limit of a surface melting process. The process may be tied to the surface melting required to explain the ages of lunar samples. Reynolds et al. (1971) have considered models for the surface heating process by a combination of accretional and radioactive means. They find certain combinations that fit the timetable of lunar rock ages. Wright (1971) suggests that the conductivity profile can be explained by oxygen depletion.

Except for the Explorer 35 survey the positive magnetic measurements of the moon have been confined to three locations for the steady field and one location for the time varying field. The successful deployment of the Apollo 15 LSM in August 1971 promises additional detail, and together with the Apollo 16 LSM will allow consideration of possible lunar asymmetry (Schubert and Schwartz, 1971a). Other refinements to be investigated are the separation of the TM and TE modes and the determination of the lunar response for various $k$ vectors.

\section{References}

Barnes, A., Cassen, P., Mihalov, J. D., and Eviatar, A.: 1971, Science 172, 716.

Behannon, K. W.: 1968, J. Geophys. Res. 73, 7257.

Blank, J. L. and Sill, W. R.: 1969, J. Geophys. Res. 74, 736.

Chapman, S. and Bartels, J.: 1962, Geomagnetism, Oxford University Press, London.

Colburn, D. S., Currie, R. G., Mihalov, J. D., and Sonett, C. P.: 1967, Science 158, 1040.

Colburn, D. S., Mihalov, J. D., and Sonett, C. P.: 1971, J. Geophys. Res. 76, 2940.

Dyal, P. and Parkin, C. W.: 1971, J. Geophys. Res. 76, 5947.

Dyal, P., Parkin, C. W., and Sonett, C. P.: 1970a, Science 169, 762.

Dyal, P., Parkin, C. W., and Sonett, C. P.: 1970b, IEEE Transactions on Geoscience Electronics GE-8, 203.

Dyal, P., Parkin, C. W., Sonett, C. P., DuBois, R. L., and Simmons, G.: 1971, in Apollo 14 Preliminary Science Report, NASA SP-272, U.S. Government, Washington, D.C.

England, A. W., Simmons, G., and Strangway, D.: 1968, J. Geophys. Res. 73, 3219.

Fricker, P. E., Reynolds, R. T., and Summers, A. L.: 1967, J. Geophys. Res. 72, 2649.

Helsley, C. E.: 1970, Science 167, 693.

Kuckes, A. F.: 1971, Nature 232, 249.

Lahiri, B. N. and Price, A. T.: 1939, Phil. Trans. Roy. Soc. A237, 509.

Mihalov, J. D., Colburn, D. S., Currie, R. G., and Sonett, C. P.: 1968, J. Geophys. Res. $73,943$.

Mihalov, J. D., Sonett, C. P., Binsack, J. H., and Moutsoulas, M. D.: 1971, Science 171, 892.

Nagata, T., Rikitake, T., and Kono, M.: 1970, in Space Research X, North Holland Publishing Co., Amsterdam.

Ness, N. F.: 1969, NASA Goddard Space Flight Center Report X-616-69-191.

Ness, N. F., Behannon, K. W., Scearce, C. S., and Cantarano, S. C.: 1967, J. Geophys. Res. $72,5769$.

Parker, E. N.: 1963, Interplanetary Dynamical Processes, Interscience, New York.

Reynolds, R. T., Fricker, P. E., and Summers, A. L.: 1971, AIAA Progress Series Volume, Lunar Thermal Characteristics, American Institute of Aeronaut ics and Astronautics, New York (in press).

Runcorn, S. K.: 1962, Nature 195, 1150.

Runcorn, S. K., Collinson, D. W., O'Reilly, W., Stephenson, A., Greenwood, N. N., and Battey, M. H.: 1970, Science 167, 697.

Schubert, G. and Colburn, D. S.: 1971, J. Geophys. Res. 76, 8174.

Schubert, G. and Schwartz, K.: 1969, The Moon 1, 106.

Schubert, G. and Schwartz, K.: 1971, Cosmic Electrodyn. 2, 244. 
Schubert, G. and Schwartz, K.: 1972, J. Geophys. Res. 77 (in press).

Schwerer, F. C., Nagata, T., and Fisher, R. M.: 1971, The Moon 2, 408.

Sill, W. R.: 1971, J. Geophys. Res. 76, 251.

Sonett, C. P.: 1965, in Space Research VI, Spartan Books, Washington, 280.

Sonett, C. P.: 1966, Minutes of Apollo Lunar Surface Science Symposium 1, E-1, unpublished.

Sonett, C. P.: 1968, IEEE Transactions on Geoscience Electronics GE-6, 126.

Sonett, C. P. and Colburn, D. S.: 1967, Nature 216, 340.

Sonett, C. P. and Mihalov, J. D.: 1972, J. Geophys. Res. 77 (in press).

Sonett, C. P. and Runcorn, S. K.: 1971, Comm. Astrophys. Space Phys. 3, 149.

Sonett, C. P., Colburn, D. S., and Schwartz, K.: 1968, Nature 219, 924.

Sonett, C. P., Colburn, D. S., Dyal, P., Parkin, C. W., Smith, B. F., Schubert, G., and Schwartz, K.: 1971a, Nature 230, 359.

Sonett, C. P., Dyal, P., Colburn, D. S., Smith, B. F., Schubert, G., Schwartz, K., Mihalov, J. D., and Parkin, C. W.: 1971b, in C. de Jager (ed.), Highlights of Astronomy 1970, D. Reidel Publ. Co., Dordrecht, p. 173.

Sonett, C. P., Dyal, P., Parkin, C. W., Colburn, D. S., Mihalov, J. D., and Smith, B. F.: 1971c, Science 172, 256.

Sonett, C. P., Schubert, G., Smith, B. F., Schwartz, K., and Colburn, D. S.: 1971d, in Proceedings of the Second Lunar Science Conference 3, MIT Press, Cambridge, Massachusetts, 2415.

Sonett, C. P., Mihalov, J. D., and Ness, N. F.: 1971e, J. Geophys. Res. 76, 5172.

Turcotte, D. L. and Oxburgh, E. R.: 1969, Nature 223, 250.

Urey, H. C., Marti, K., Hawkins, J. W., and Liu, M. K.: 1971, in Proceedings of the Second Lunar Science Conference 2, MIT Press, Cambridge, Massachusetts, 987.

Wright, D. A.: 1971, Nature Phys. Sci. 231, 169.

Wilcox, J. M. and Colburn, D. S.: 1970, J. Geophys. Res. 75, 6366. 